\title{
Replication of Functional MRI Detection of Deception
}

\author{
F. Andrew Kozel*,1,2, Steven J. Laken ${ }^{3}$, Kevin A. Johnson ${ }^{2}$, Bryant Boren ${ }^{1}$, Kimberly S. Mapes ${ }^{1}$, \\ Paul S. Morgan ${ }^{2,4}$, and Mark S. George ${ }^{2}$
}

${ }^{I}$ Department of Psychiatry, University of Texas Southwestern Medical Center, Dallas, Texas, USA; ${ }^{2}$ Center for Advanced Imaging Research (CAIR), Medical University of South Carolina Charleston, South Carolina, USA; ${ }^{3}$ Cephos Corp., Pepperell, Massachusetts, USA ${ }^{4}$ Academic Radiology, University of Nottingham, Nottingham, United Kingdom

\begin{abstract}
Background: Several studies support the use of fMRI for detecting deception. There have been, however, no reported replications on different scanners or at different locations. In a previous study, deception was accurately detected in at least $90 \%$ of the participants in two independent cohorts. This study attempted to replicate those findings using a different scanner and location.

Methods: Healthy participants 18-50 years of age were recruited from the local community. After providing written informed consent, participants were screened to ensure that they were healthy, not taking any medications, and safe to have an MRI. For the testing paradigm, subjects chose one of two objects (ring or watch) to "steal" and placed it in their locker. Participants were then scanned while being visually presented with a series of questions. Functional MRI analysis was performed in the same manner as described in Kozel et al. 2005. A Chi-Squared test was used to test for a significant difference between the results in the previous study and in this replication study.

Results: Thirty subjects (20 women, mean age 29.0 6.5 years) were scanned with one subject being noncompliant with the protocol. Twenty-five of twenty-nine (86\%) participants were correctly identified when being deceptive. There was no statistical difference between the accuracy rate obtained in this study (25/29) versus the previous study (28/31) (ChiSquared, $\left.\chi^{2}=0.246, \mathrm{p}=0.6197\right)$.

Conclusions: Our methodology for detecting deception was successfully replicated at a different site suggesting that this methodology is robust and independent of both scanner and location.
\end{abstract}

Keywords: fMRI, Individual analysis, lie detection, mock crime, neuroimaging, and replication.

\section{INTRODUCTION}

More accurate methods to detect deception are currently needed [1]. Most presently available technologies to detect deception such as the polygraph, thermal imaging, and voice stress analyzers actually detect arousal and not deception. In an attempt to develop a technology that is more reliable, recent investigations have focused on detecting deception itself in the brain where the lies are generated [2-20] (for review see [21]).

After successfully replicating the group results of fMRI detection of deception in a laboratory paradigm involving subjects giving deceptive and truthful answers [3, 4], we developed a method to identify deception at the individual level [5]. In the original method building study performed at the Medical University of South Carolina, we recruited a sample of thirty subjects (Model Building Group) in which to build an analysis model of detecting deception at the individual level. Participants were instructed to steal a watch or a ring and place it in their locker. While being scanned inthe MRI, they responded to visually presented questions. Participants were given an incentive to conceal which object

*Address correspondence to this author at the UT Southwestern Medical Center, Department of Psychiatry, 5323 Harry Hines Blvd. Dallas, TX 75390-9119, USA; Tel: 1-214-648-0157; Fax: 1-214-648-0168;

E-mail: Andrew.Kozel@utsouthwestern.edu they took and respond as though they took neither object. Using the data from these thirty subjects, an analysis model was designed that accurately detected deception for $93 \%$ of the subjects. Since we used the data to build the model, we recruited an independent group of thirty-one participants to test the model. In this "Model Testing Group," deception was detected accurately for $90 \%$ of the participants.

Since both cohorts were scanned on the same 3 Tesla $(\mathrm{T})$ Philips Intera MR scanner and were drawn from the same population (Charleston, SC), whether the results were scanner or location dependent was not known. Testing the same participants using motor and/or sensory tasks on various scanners has demonstrated that fMRI results can be consistent across different scanner [22, 23]. There are, however, few examples in the literature of individual results in cognitive tasks using fMRI $[24,25]$. Whether the prior testing model would hold true for a cognitive task on a different scanner with a different group of participants required investigation. Thus, we tested the same protocol at UT Southwestern Medical Center in Dallas (UTSW) on a 3T Philips Achieva to determine if we could replicate the previous findings.

\section{MATERIALS AND METHODOLOGY}

Complete details of the current study protocol are published in prior work [5]. We did not vary from the core 
methods used in that study. Healthy, non-medicated participants age 18-50 were recruited from the local community. After obtaining written informed consent as approved by the University of Texas Southwestern Medical Center at Dallas Investigational Review Board, participants were screened with a Structured Clinical Interview for DSMIV Axis I Disorders (SCID-I) [26], a pre-MRI screening form, a medical history, a physical exam, and an Annette Handedness Scale [27]. A urine sample was obtained for a drug urinalysis and a urine pregnancy test (if the participant was a woman with child bearing potential). After the screening was completed, eligible participants were scheduled for the fMRI testing portion of the study. Also, the questions to be asked in the scanner were reviewed with the subjects.

On the day of scanning, participants were again screened to ensure that they were safe to enter the MR environment and the instructions were reviewed. The only investigators present for the scanning were KSM and FAK. The participants were told to go to another room by KSM and steal one of two objects in a drawer and place it in their locker. After the subjects performed the task, they then practiced the questions on a computer outside of the scanner with KSM present but with FAK absent. Thus KSM ensured that the participants carried out the procedure properly, and FAK was experimentally blinded to which object was taken. For the deception task, four types of questions were visually displayed to subjects: "ring" - regarding whether they took the ring; "watch" - regarding whether they took the watch; "neutral" - general questions with clear yes and no answers; and "control" - questions about doing minor wrongful acts. Slight changes in the wording for several neutral and control questions were required because of the different imaging site. Participants were instructed to answer the ring and watch questions as if they had not stolen either object and to answer the control and neutral questions truthfully. Subjects were given the incentive that they would receive an additional \$50 if investigator FAK could not tell when they were lying in the scanner. This provided motivation for subjects to attempt countermeasures when lying. All subjects received the additional $\$ 50$.

After participants completed the practice, they were placed in the MR scanner with foam head packing and ear protection. Scanning was performed with a 3T Philips Achieva (Philips Medical System, The Netherlands) at UTSW using an eight-channel SENSE head coil. Subjects performed a simple Motor task (6 minutes), then the Deception task (16 minutes), and finally a T1-weighted structural scan. For the Deception task, 497 echoplanar imaging (EPI) transverse images (TR $1934 \mathrm{~ms}$, TE 35 ms, Flip Angle 90 deg, FOV 208 mm, matrix 64x64, SENSE factor 2, 36 slices, $3 \mathrm{~mm}$ with $0 \mathrm{~mm}$ gap, giving a voxel size of $3.25 \times 3.25 \times 3.00$ $\mathrm{mm}^{3}$ ) were acquired that covered the entire brain and were positioned with reference to the anterior commissureposterior commissure (AC-PC) line using a sagittal scout image. Most scanning parameters were the same from the previous study except that the TR (1934 versus $1867 \mathrm{~ms}$ ) and TE (35 versus $30 \mathrm{~ms}$ ) were slightly longer resulting in fewer volumes (497 versus 515).

The questions were presented in a pseudorandomized order and the responses were recorded using E-Prime (Psy- chology Software Tools, Inc. Pittsburgh, PA). Using a custom built apparatus (MRA Incorporated, Washington, PA), questions were visually displayed to the subjects using a rear projection system and button press signals were transmitted to a computer outside the scanner room. There were 20 questions in each category and each question was asked twice for a total of 160 events. The questions were presented visually for 3.5 seconds. After a question, there was a visual prompt for the subject to answer "Yes or No" for 2 seconds followed by a "+" for 0.5 seconds. The total time per question event was six seconds. Subjects were instructed not to answer until they saw the visual prompt of "Yes or No". The delayed response was to reduce the variability of response timing due to differences in reading speeds across questions and across subjects. After scanning, subjects completed a questionnaire about the study.

Functional MRI analysis was carried out using Statistical Parametric Mapping software (SPM 2, Wellcome Department of Cognitive Neurology, London, UK) and Matlab version 2006a by two investigators (SJL and FAK) independently. The two analyses differed in the following ways; FAK performed SPM2 analysis using Red Hat Linux Enterprise Edition (Linux kernel 2.6) and SJL performed SPM2 analysis using Windows XP x64. The analysis was performed in the same manner as in Kozel et al. 2005 except that automated Matlab scripts were written (by SJL) for consistency in analysis. Importantly, with use of automated scripts, the only judgment required of the person performing the data analysis was to identify the approximate location of the anterior commissure prior to processing. Briefly, the functional imaging data were reoriented to the anterior commissure, realigned and unwarped, slice timing corrected, spatially normalized and spatially smoothed. Individual tmaps were generated for the contrasts Ring-minus-Neutral and Watch-minus-Neutral. The three regions of interest identified in Kozel et al. 2005 were applied to these two contrasts for each individual subject. The contrast with the greater number of activated voxels $(\mathrm{p} \leq 0.05)$ was determined and identified the object about which the participant was being deceptive. All data were checked for quality regarding completing the protocol properly, adequate number of behavioral responses, lack of significant image artifact, lack of excessive motion (greater than $3 \mathrm{~mm}$ ), and differences in calls by FAK and SJL.

The group map analysis of lie minus true was evaluated to determine if prior regions of significant (FDR $\leq 0.05$, required number of activated voxels in a cluster $(\mathrm{K}) \geq 25$ ) activation replicated from previous studies. Using the individual Lie-minus-True and True-minus-Lie contrast images produced at the first statistical level analysis, group t-maps were generated at the second level using a random effects model [28].

\section{RESULTS}

Thirty-three subjects were enrolled with three not being scanned (two with prior history of mood disorder, and one failed to return for the scan). Of the 30 subjects scanned (20 women, mean age $29.0 \pm 6.5$ years), one failed a quality check by taking a medication the night before he was scanned (imaged but not included into the data analysis). The 
remaining 29 participants passed all quality checks, including having the exact same determination of when they were being deceptive by the two investigators independently performing the analysis. Using the a priori defined methodology, 25/29 (86\%: 95\% CI 0.68 - 0.96) participants were correctly identified when being deceptive (significantly different from chance, one-tailed Exact Binomial, $\mathrm{z}=5.4$, $\mathrm{p}<0.000001)$. There was no statistical difference between the blind testing accuracy rate obtained in this study at UTSW $(25 / 29)$ versus the blind testing at MUSC (28/31) (ChiSquared, $\left.\chi^{2}=0.246, \mathrm{p}=0.6197\right)$ which indicates that this methodology is likely largely independent of scanner and location.

The Lie-minus-True group map revealed significant activation in similar regions from the prior cohorts using this paradigm (see Figure and Table). Specifically, the regions of right orbitofrontal, right inferior frontal, right middle frontal, and right cingulate gyrus have replicated now in five independent groups from four studies across several paradigms [3-5]. Interestingly, the left middle temporal gyrus was not significantly active in this study as it had been in the prior three studies, but is not included in the model to detect deception at the individual level. The True-minus-Lie group map revealed no significant activation.

\section{DISCUSSION}

This method for detecting deception was successfully replicated on a different scanner at a different location for both the individual and group analyses. This suggests that our deception detection methodology is neither scanner nor location dependent. To our knowledge, this is the first replication at the individual level for fMRI detection of deception using a different scanner. Successful replication is a critical step in the development phase of a functional neuroimaging diagnostic test [29].

In a fMRI detection of deception study performed by our group using a mock sabotage crime paradigm, a similar version of the ring-watch testing was performed as a study was to determine if the ring-watch paradigm could be used as an "internal control", to predict which participants had valid mock-crime determinations. However, we noted a

A

Model Building Group (Kozel et al., 2005): Lie-minus-True, n= 30

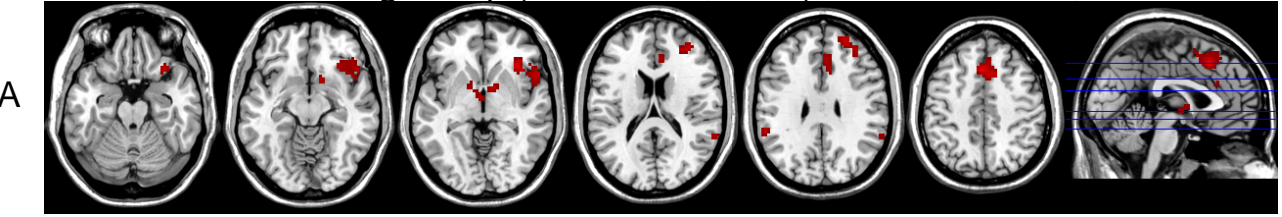

ROls from Model Building Group: Lie-minus-True

$\mathrm{B}$

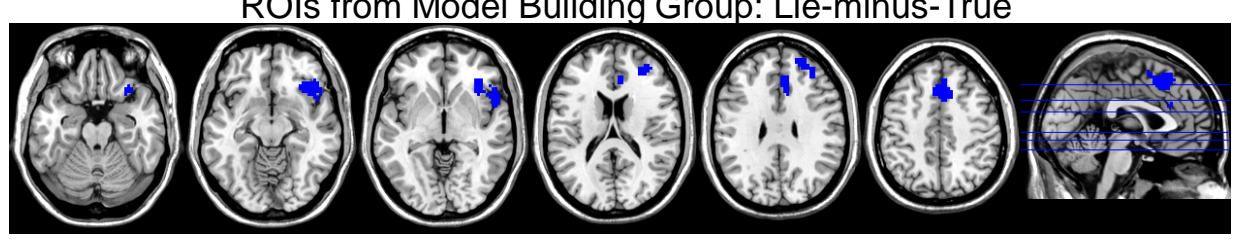

UT Southwestern Group: Lie-minus-True, $n=29$

C

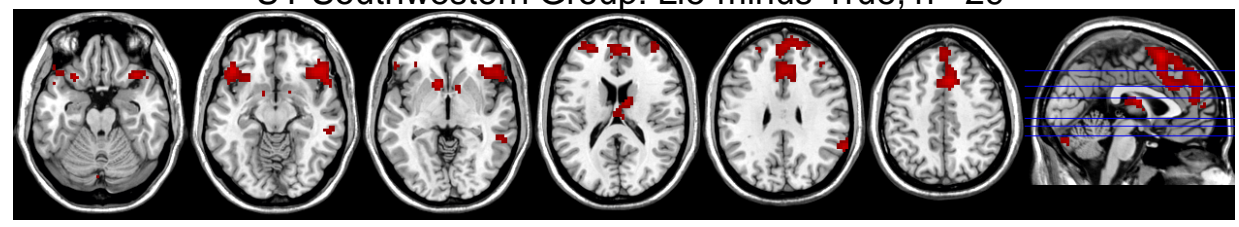

UT Southwestern Group with ROIs: Lie-minus-True, $n=29$

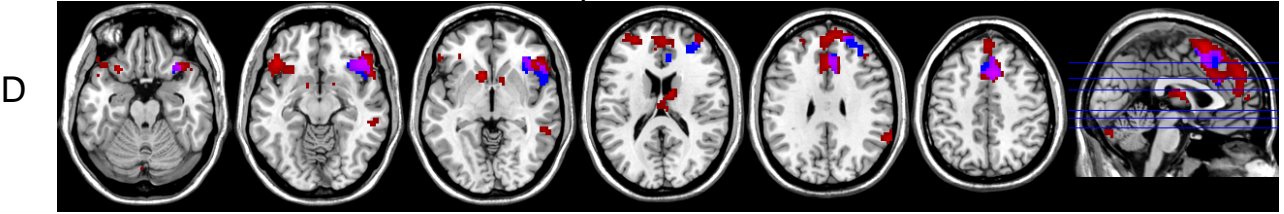

Fig. (1). Comparison of Lie-Minus-True Group Maps

The Model Building Group results of lie-minus-true were used to determine the regions of interest for the individual analysis methodology (see red regions in row A). Three of the clusters were designated as regions of interest (ROI) for the individual analyses (see blue regions in row B - complete details in Kozel et al. 2004). The UT Southwestern Group results for the contrast lie-minus-true are presented using the same statistical threshold (FDR $\leq 0.05, \mathrm{k} \geq 25$ ) and image slices in Row C. Row D indicates the overlap of the UT Southwestern Group results and the a priori determined ROIs from the Model Building Group. The regions indicated in red correspond to area with significant activation (FDR $\leq 0.05, \mathrm{k} \geq 25$ ) from the UT Southwestern Group. The voxels corresponding to the ROIs are indicated with blue. The voxels in which both the activation and regions of interest overlap are indicated with purple. Using MRIcron (http://www.sph.sc.edu/comd/rorden/mricron/) significant areas of activation (FDR $\leq 0.05, \mathrm{k} \geq 25$ ) and/or ROIs for the respective groups are overlaid on a structural template image. The axial images of the structural template are arranged from ventral to dorsal. The sagittal images to the right of the figure specify the levels of the axial slices. The $\mathrm{z}$ levels for all figures are $-21,-11,-3,18,30$, and 46 from left to right of the image. The right of the axial brain image is the right side of the brain. Similarities can be seen between the activation pattern in the Model Building group and the UT Southwestern Medical Center group. 
Table UT Southwestern - Group Results Lie-Minus-True

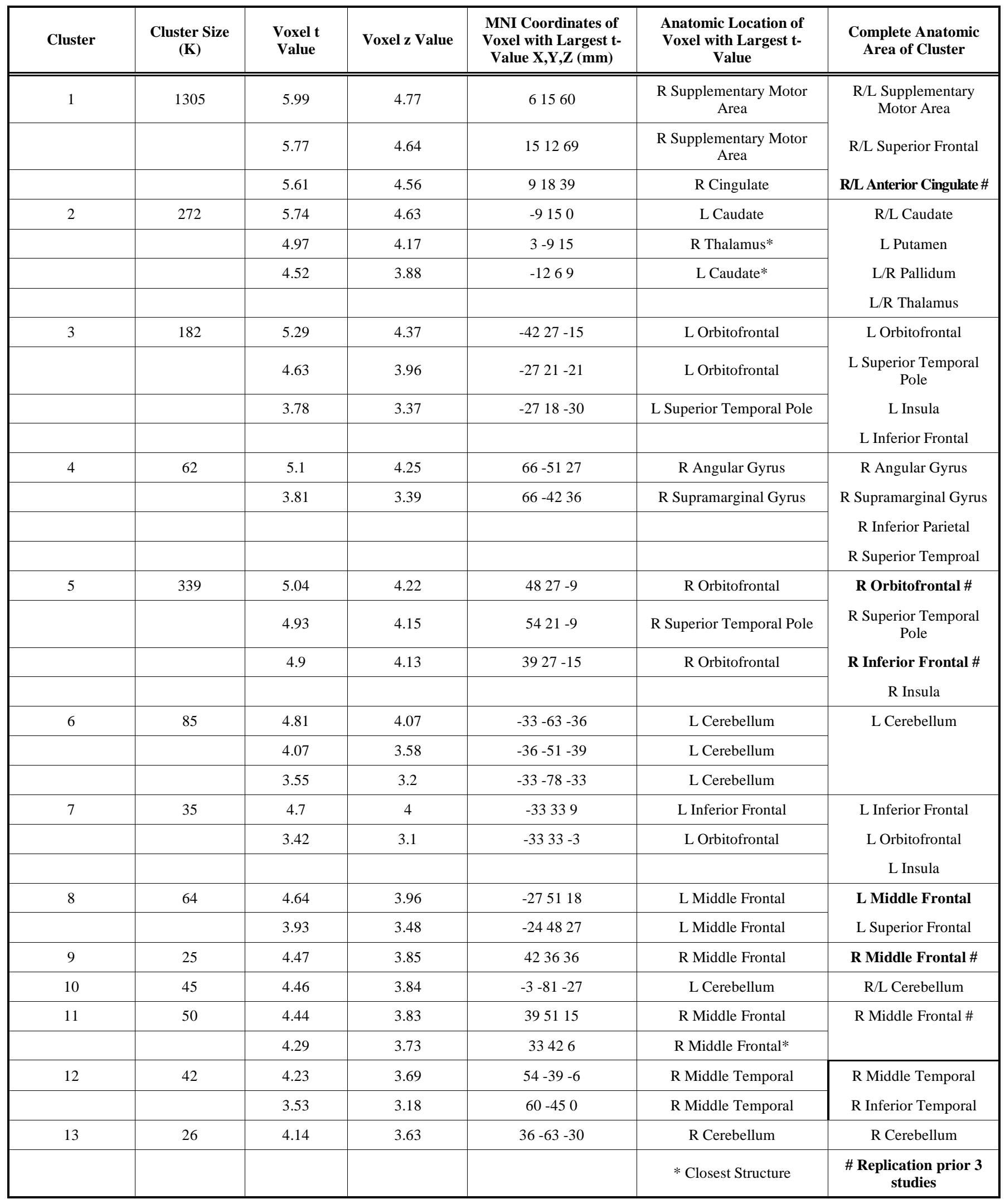

validation step [30]. The primary objective of this mockcrime testing study, in which 57 participants were scanned, was to determine whether or not participants had committed a mock-sabotage crime. After testing the participants in the scanner regarding the mock-crime, they also completed the ring-watch testing paradigm. A secondary objective of the 
decrease in accuracy of this internal control ring-watch paradigm $(71 \%)$. This is in contrast to the accuracy results of our prior two groups, $93 \%$ and $90 \%$ [5], as well as this current study with an accuracy of $86 \%$. The ring-watch paradigm used in the mock-crime study had significant differences from those in the original ring-watch testing study and this study. In the mock-crime study, to make the scanning more time efficient, only the three categories of questions (ring, watch, neutral) used in the analysis were presented to subjects. The control questions were omitted. Although this reduced scanning time, it reduced the power of the testing paradigm to detect differences. This reduced paradigm power may have contributed to the lower accuracy rates in the mock-crime ring-watch testing. Interestingly, the same reduction in question number was made to the mockcrime testing paradigm. With this reduced power, the technology was found to be highly sensitive (100\%) but low on specificity (33\%) [30]. Further testing can determine if increasing the power of the questioning format can improve the accuracy rates.

There are several other discrepancies between the ringwatch testing in the mock-crime study and the previous and current study. For the mock-crime testing, participants were put through the ring-watch testing only after undergoing testing for what was the primary focus of the study, the mock-crime testing. In addition, there was no monetary incentive offered to "fool" a researcher in the ring-watch testing. Thus, fatigue and lack of motivation may have impacted the results. Future testing should address these important issues to determine what impact if any they have on fMRI detection of deception.

An important issue in any test development is the potential impact of bias. A strength of this methodology is the automated analysis that drastically reduces any chance for bias. The only decision required of the person performing the data analysis is to approximately locate the anterior commissure on the brain image. In this study, two investigators performed the analysis independently and arrived at the same results. One investigator, FAK, was aware of which object the subject took at the time of scanning. However, the other investigator, SJL, never saw any of the participants, knew nothing about them, and did not know which object they had taken prior to making his determination of when they were being deceptive. Thus, these aspects of the methodology and independently confirmed results support the minimal impact that bias had on the outcome.

Although this is an important validation step, further work needs to address how robust these findings might be with different testing scenarios and populations. The scenario used was a simple laboratory paradigm with healthy adult participants. Testing when there is greater risk (e.g. prison, large financial loss, etc.) or in people with illnesses taking medications may result in a different outcome. Another important consideration is that this study only provides support for the analysis methodology used in these studies. Different analysis strategies and testing formats will require independent evaluation and replication.

\section{CONCLUSION}

In conclusion, this study provides further support for the feasibility of using fMRI to detect deception and demonstrates that the initial findings are location, cohort, and scanner independent.

\section{CONFLICTS OF INTEREST}

In terms of disclosure, the Medical University of South Carolina, in conjunction with Drs. Kozel and George, has a pending patent application for using fMRI to detect deception. Drs. Kozel and George have no equity investment in this work other than their role as co-inventors. Dr. Laken is the President and CEO of Cephos Corp. and holds stock in the company. Cephos Corp. has exclusively licensed the MUSC patent applications and the Medical University of South Carolina Foundation for Research Development holds stock in Cephos Corp. Dr. Kozel has received research support from Defense Agency for Credibility Assessment (DACA) and Cephos Corp. Drs. Johnson and George have received salary support for DACA. We do not think that this patent application or funding has in any way impacted on this study, or its interpretation. Nevertheless, it is important for reviewers and readers to be aware of this potential conflict.

\section{ACKNOWLEDGMENTS}

Funding provided by Department of Defense Polygraph Institute (W74V8H-04-1-0010). Grant-in-kind support provided by Cephos Corp. Dr. Kozel was supported by a K23 from the NIMH (5K23MH070897-01). The Advanced Imaging Research Center at UT Southwestern Medical Center provided grant-in-kind support for MR scanning. We wish to thank Mr. Dave Ramsey and Mr. John Dornisch of the South Carolina Research Authority for computing support; Ms Rani Varghese for MR scanning; and Ms Michelle Waller-George for administrative support. The views expressed in this article are those of the authors and do not necessarily reflect the official policy or position of the Department of Defense, the National Institute of Mental Health, the National Institutes of Health, or the U.S. Government.

\section{OTHER PRESENTATIONS OF THIS WORK}

1) A poster was presented at the American College of Neuropsychopharmacology meeting in Boca Rotan, Dec 2007.

2) A portion of this work was presented at the National Judicial College on November 14, 2007.

\section{REFERENCE}

[1] National Research Council, The Polygraph and Lie Detection Committee to Review the Scientific Evidence on the Polygraph. Division of Behavioral and Social Sciences and Education. The National Academies Press: Washington, DC, 2003.

[2] Ganis, G.; Kosslyn, S. M.; Stose, S.; Thompson, W. L.; YurgelunTodd, D. A. Neural correlates of different types of deception: an fMRI investigation. Cereb. Cortex, 2003, 13(8), 830-6.

[3] Kozel, F. A.; Revell, L. J.; Lorberbaum, J. P.; Shastri, A.; Elhai, J. D.; Horner, M. D.; Smith, A.; Nahas, Z.; Bohning, D. E.; George, M. S. A pilot study of functional magnetic resonance imaging brain correlates of deception in healthy young men. J. Neuropsychiatr. Clin. Neurosci., 2004, 16(3), 295-305. 
[4] Kozel, F. A.; Padgett, T. M.; George, M. S. A replication study of the neural correlates of deception. Behav. Neurosci., 2004, 118(4), 852-6.

[5] Kozel, F. A.; Johnson, K. A.; Mu, Q.; Grenesko, E. L.; Laken, S. J.; George, M. S. Detecting deception using functional magnetic resonance imaging. Biol. Psychiatry, 2005, 58(8), 605-13.

[6] Spence, S. A.; Farrow, T. F.; Herford, A. E.; Wilkinson, I. D.; Zheng, Y.; Woodruff, P. W., Behavioural and functional anatomical correlates of deception in humans. Neuroreport, 2001, 12(13), 2849-2853.

[7] Langleben, D. D.; Schroeder, L.; Maldjian, J. A.; Gur, R. C.; McDonald, S.; Ragland, J. D.; O'Brien, C. P.; Childress, A. R., Brain activity during simulated deception: an event-related functional magnetic resonance study. Neuroimage, 2002, 15(3), 727-32.

[8] Faro, S.; Mohamed, F.; Gordon, N.; Platek, S.; Williams, M.; Ahmad, H. Functional MRI of Deception and Truth with Plolygraph Correlation. RSNA annual meeting 2004, Abstract.

[9] Mohamed, F. B.; Faro, S. H.; Gordon, N. J.; Platek, S. M.; Ahmad, H.; Williams, J. M., Brain mapping of deception and truth telling about an ecologically valid situation: functional MR imaging and polygraph investigation--initial experience. Radiology, 2006, 238(2), 679-88

[10] Langleben, D. D.; Loughead, J. W.; Bilker, W. B.; Ruparel, K.; Childress, A. R.; Busch, S. I.; Gur, R. C. Telling truth from lie in individual subjects with fast event-related fMRI. Hum. Brain Mapp., 2005, 26(4), 262-72.

[11] Davatzikos, C.; Ruparel, K.; Fan, Y.; Shen, D. G.; Acharyya, M.; Loughead, J. W.; Gur, R. C.; Langleben, D. D. Classifying spatial patterns of brain activity with machine learning methods: application to lie detection. Neuroimage, 2005, 28(3), 663-8.

[12] Abe, N.; Suzuki, M.; Mori, E.; Itoh, M.; Fujii, T. Deceiving others: distinct neural responses of the prefrontal cortex and amygdala in simple fabrication and deception with social interactions. J. Cogn. Neurosci., 2007, 19(2), 287-95.

[13] Abe, N.; Suzuki, M.; Tsukiura, T.; Mori, E.; Yamaguchi, K.; Itoh, M.; Fujii, T. Dissociable roles of prefrontal and anterior cingulate cortices in deception. Cereb. Cortex, 2006, 16(2), 192-9.

[14] Nunez, J. M.; Casey, B. J.; Egner, T.; Hare, T.; Hirsch, J. Intentional false responding shares neural substrates with response conflict and cognitive control. Neuroimage, 2005, 25(1), 267-77.

[15] Lee, T. M.; Liu, H. L.; Tan, L. H.; Chan, C. C.; Mahankali, S.; Feng, C. M.; Hou, J.; Fox, P. T.; Gao, J. H. Lie detection by functional magnetic resonance imaging. Hum. Brain Mapp., 2002, 15(3), 157-64.

[16] Lee, T. M.; Liu, H. L.; Chan, C. C.; Ng, Y. B.; Fox, P. T.; Gao, J. H. Neural correlates of feigned memory impairment. Neuroimage, 2005, 28(2), 305-13.
[17]

Phan, K. L.; Magalhaes, A.; Ziemlewicz, T. J.; Fitzgerald, D. A.; Green, C.; Smith, W. Neural correlates of telling lies: a functional magnetic resonance imaging study at 4 tesla. Acad. Radiol., 2005, $12,164-172$.

[18] Kozel, F. A.; Johnson, K. A.; Mu, Q.; Grenesko, E. L.; Laken, S. J.; Smith, J.; Walker, J.; George, M. S. Can simultaneously acquired electrodermal activity improve accuracy of fMRI detection of deception? Social Neurosci., 2007, In Press.

[19] Spence, S. A.; Kaylor-Hughes, C.; Farrow, T. F.; Wilkinson, I. D. Speaking of secrets and lies: The contribution of ventrolateral prefrontal cortex to vocal deception. Neuroimage, 2008, 40(3), 1411-8.

[20] Abe, N.; Okuda, J.; Suzuki, M.; Sasaki, H.; Matsuda, T.; Mori, E.; Tsukada, M.; Fujii, T. Neural Correlates of True Memory, False Memory, and Deception. Cereb Cortex, 2008.

[21] Johnson, K. A.; George, M. S.; Kozel, F. A. Detecting deception using functional magnetic resonance imaging. Direc. Psychiatry, 2007, 27, In Press.

[22] Friedman, L.; Glover, G. H.; Krenz, D.; Magnotta, V. Reducing inter-scanner variability of activation in a multicenter fMRI study: role of smoothness equalization. Neuroimage, 2006, 32(4), 165668.

[23] Costafreda, S. G.; Brammer, M. J.; Vencio, R. Z.; Mourao, M. L.; Portela, L. A.; de Castro, C. C.; Giampietro, V. P.; Amaro, E., Jr Multisite fMRI reproducibility of a motor task using identical MR systems. J. Magn. Reson. Imaging, 2007, 26(4), 1122-6.

[24] Bush, G.; Shin, L. M.; Holmes, J.; Rosen, B. R.; Vogt, B. A. The Multi-Source Interference Task: validation study with fMRI in individual subjects. Mol. Psychiatry, 2003, 8(1), 60-70.

[25] Bush, G.; Shin, L. M. The Multi-Source Interference Task: an fMRI task that reliably activates the cingulo-frontal-parietal cognitive/attention network. Nat. Protoc., 2006, 1(1), 308-13.

[26] First, M.; Spitzer, R.; Williams, J.; Gibbon, M. Structured clinical interview for DSM-IV (SCID). American Psychiatric Press: Washington, D.C., 1995.

[27] Annette, M. A classification of hand preference by association analysis. Br. J. Psychol., 1970, 61, 303-321.

[28] Friston, K. J.; Frackowiak, R. S. J. Images of the future. In Human brain function, Frackowiak, R. S. J.; Friston, K. J.; Dolan, R. J.; Frith, C. D.; Mazziotta, J. C., Eds. Academic Press: San Diego, CA, 1997; pp. 487-517.

[29] Kozel, F. A.; Trivedi, M. H. Developing a neuropsychiatric functional brain imaging test. Neurocase, 2008, 14(1), 54-58.

[30] Kozel, F.; Johnson, K.; Grenesko, E.; Laken, S.; Kose, S.; Lu, X.; Pollina, D.; Ryan, A.; George, M. Functional MRI Detection of deception after committing A Mock Sabotage Crime. J. Forensic Sci., 2009, in press.

Received: August 04, 2008

Revised: October 03, 2008

Accepted: October 24, 2008

() Kozel et al:; Licensee Bentham Open.

This is an open access article licensed under the terms of the Creative Commons Attribution Non-Commercial License (http://creativecommons.org/licenses/by-nc/3.0/) which permits unrestricted, non-commercial use, distribution and reproduction in any medium, provided the work is properly cited. 